\title{
Caudatin induces cell apoptosis in gastric cancer cells through modulation of Wnt/ $\beta$-catenin signaling
}

\author{
XIAOFENG LI ${ }^{1 *}$, XIAOTING ZHANG ${ }^{1 *}$, XIZHI LIU $^{1 *}$, ZHIWEN TAN $^{1}$, CELI YANG $^{1}$, XIAOFENG DING ${ }^{1,2}$, \\ XIANG HU ${ }^{1,2}$, JIANLIN ZHOU ${ }^{1}$, SHUANGLIN XIANG ${ }^{1,2}$, CHANG ZHOU $^{1,2}$ and JIAN ZHANG ${ }^{1}$ \\ ${ }^{1}$ Key Laboratory of Protein Chemistry and Developmental Biology of the State Education Ministry of China, \\ College of Life Science, Hunan Normal University, Changsha, Hunan 410081; \\ ${ }^{2}$ Hunan Institute of Biology, Changsha, Hunan 410006, P.R. China
}

Received March 11, 2013; Accepted April 16, 2013

DOI: $10.3892 /$ or.2013.2495

\begin{abstract}
Caudatin has been reported to trigger apoptosis in several types of cancer cell lines. In the present study, we investigated whether caudatin has therapeutic value in gastric cancer and examined the effects of caudatin on the expression of $\beta$-catenin in human gastric carcinoma cell lines. Here, we showed that caudatin treatment resulted in a dose- and time-dependent inhibition of proliferation of the gastric carcinoma cell lines. Cell cycle analysis demonstrated that caudatin induced G0/G1 arrest and downregulated CDK2 levels. In contrast, the expression of the p21 protein was increased. AGS cells treated with caudatin exhibited typical characteristics of apoptosis, which were accompanied by activation of caspase-3, $-8,-9$ and PARP. Western blot analysis and immunocytochemical staining showed that caudatin induced a reduction in $\beta$-catenin expression and this reduction was due to proteosome-mediated degradation. This reduction in $\beta$-catenin activation was due to the downregulation of its downstream targets cyclinD1 and c-MYC in all gastric carcinoma cell lines. Furthermore, we demonstrated that gastric adenocarcinoma tissues and AGS cells exhibited abnormal expression of miR-372. Additionally, caudatin downregulated the expression of oncomir miR-372 and miR-21, and upregulated tumor suppressor let-7a miRNA expression. These data revealed that inhibition of $\mathrm{Wnt} / \beta$-catenin signaling is a novel mechanism of action for caudatin during therapeutic intervention in gastric cancers.
\end{abstract}

\section{Introduction}

Gastric cancer is one of the most common malignancies and the second leading cause of cancer-related mortality worldwide.

Correspondence to: Dr Chang Zhou, Key Laboratory of Protein Chemistry and Developmental Biology of the State Education Ministry of China, College of Life Science, Hunan Normal University, 36 Lushan Road, Changsha, Hunan 410081, P.R. China

E-mail: zhouchang1972@126.com

*Contributed equally

Key words: caudatin, $\beta$-catenin, cell apoptosis, gastric cancer cells, miRNA
Surgery and systemic treatment regimens in combination with radiotherapy and chemotherapy are able to cure this malignancy in the majority of cases. Unfortunately, the young age of typical gastric cancer patients further enhances the time at risk for the side effects of these therapies. Delayed toxicity and the development of secondary malignancies are a serious concern for the current anti-neoplastic armamentarium $(1,2)$. Therefore, it is urgent to further characterize the entity of gastric tumors to better understand and predict their biological behaviors and to identify novel high efficient and low toxic therapeutic agents for the treatment of gastric cancer in clinical practice (3).

Modern pharmacologic study proves that C-21 steroidal glycosides are important biological active compounds, which are widely found in the plants of the Asclepiadaceae family and exhibit extensive pharmacological effects such as inhibition of proliferation, induction of apoptosis and inhibition of the invasion of tumor cells $(4,5)$. Caudatin, a C-21 steroidal glycoside, is mainly isolated from the root of Cynanchum bungei Decne in China. Recent studies demonstrated that caudatin induced the apoptosis of HepG2 cells or the SMMC772 cell line $(6,7)$. Wang et al (8) found that caudatin had an inhibitory activity on the secretion of HBsAg and HBV DNA replication. Furthermore, caudatin as a prospective anti-HCC drug with the mechanism of inhibiting cell proliferation and inducing cell apoptosis has been reported (9). Yet, there is no report concerning the effects of caudatin on gastric adenocarcinoma, and the underlying mechanisms are not well documented.

The Wnt/ $\beta$-catenin signaling pathway is involved in multiple developmental events during embryogenesis and is also implicated in tumorigenesis, cell-fate decisions, proliferation and apoptosis $(10,11)$. Wnt ligands trigger at least three different intracellular signaling cascades: the canonical Wnt pathway, which results in transcriptional regulation of target genes via $\beta$-catenin; the planar cell polarity (PCP) pathway; and the Wnt-dependent calcium/protein kinase C (PKC) pathway. The protein $\beta$-catenin is the central player in one major arm of the canonical Wnt pathway $(12,13)$. Hyperactivation of $\beta$-catenin signaling has been implicated as a driver of various types of cancers, including human gastric cancer (14-16). Thus, there is a great interest in identifying inhibitors of the $\mathrm{Wnt} / \beta$-catenin signaling pathway. 
miRNAs are small non-coding RNAs consisting of $\sim 17-24$ nucleotides, each of which is predicted to regulate hundreds of genes (both coding and non-coding) by posttranscriptional silencing (17). Many have been shown to function in cell proliferation, apoptosis, migration and development $(18,19)$. Certain miRNAs have been reported to be associated with the initiation and progression of gastric cancer. For example, aberrant expression of miR-106a was detected in gastric carcinoma tissue and was found to promote gastric carcinogenesis (20). miR-21 has been found to be correlated with $H$. pylori infection and gastric cancer progression (21). Recently, Belair et al (22) studied the miRNA expression profile of AGS cells and found that miR-372 had the highest expression of the total miRNAs with the percentage of miR-372 reaching $11 \%$. We previously reported that miR-372 maintains oncogene characteristics by targeting TNFAIP1-regulated AGS cell growth (23). Moreover, recent studies have demonstrated a connection between miRNAs and the $\mathrm{Wnt} / \beta$-catenin signaling pathway. Zhou et al (24) reported that $\beta$-catenin transactivates the microRNA-371-373 cluster in colorectal cancer (CRC) cell lines. In contrast, the let-7 microRNA family was found to be downregulated in human gastric cancer $(25,26)$. These findings demonstrate that miRNAs are involved in gastric carcinogenesis via varied patterns and pathways.

In the present study, we evaluated the effects of caudatin on gastric cancer cells and elucidated the mechanism mediated through the Wnt/ $\beta$-catenin signaling pathway.

\section{Materials and methods}

Reagents. Caudatin was purchased from the Shenzhen Medherb Biotechnology Co., Ltd. (Shenzhen, China) and dissolved with $100 \%$ dimethyl sulfoxide (DMSO) (concentration of the stock solution, $10 \mathrm{mM}$ ).

Human tissue samples. All human gastric adenocarcinoma tissue samples and adjacent normal tissues were obtained from the Department of Pathology, Xiangya Hospital, Changsha, China. The samples were classified according to World Health Organization criteria published in 2000. All patients signed consent forms and the study protocol was approved by the Committee on Human Rights in Research of the Ethics Committee of the College of Life Science, Hunan Normal University, Changsha, China.

Cells and cell culture. The human gastric carcinoma cell lines (AGS, HGC-27, GES-1) were obtained from the Cell Bank of the Chinese Academy of Sciences (China). Cells were cultured at $37^{\circ} \mathrm{C}$ in $5 \% \mathrm{CO}_{2}$ in $\mathrm{F} 12$ or Dulbecco's modified Eagle's medium (DMEM; both from Gibco-BRL) supplemented with $10 \%$ fetal bovine serum (Gibco-BRL), $100 \mathrm{U} / \mathrm{ml}$ penicillin and $100 \mu \mathrm{g} / \mathrm{ml}$ streptomycin.

MTT assay. Briefly, cells were seeded in 24-well tissue culture plates in a final volume of $500 \mu \mathrm{l}$. After attachment for $24 \mathrm{~h}$, cells were treated with $25-100 \mu \mathrm{M}$ caudatin or DMSO as a blank. After drug exposure, $50 \mu \mathrm{l}$ of MTT [5 mg/ml in phosphate-buffered saline (PBS)] solution was added to each well for an additional $4 \mathrm{~h}$. The reaction was stopped by removal of MTT, and the formazan crystals were solubilized in $100 \mu \mathrm{l}$
DMSO (Sigma, St. Louis, MO, USA) in each well. Absorbance at $570 \mathrm{~nm}$ was recorded using a microplate spectrophotometer. Three wells were assigned to each caudatin dose.

FACS assays. Cells were harvested at $600 \mathrm{x} \mathrm{g}$ for $3 \mathrm{~min}$, washed twice in PBS at room temperature and resuspended in appropriate PBS. Then, cells were resuspended in 1X FACS binding buffer containing Annexin $\mathrm{V}$ and propidium iodide and analyzed using a FACS flow cytometer (BD Biosciences). A total of 20,000 cells was counted for each sample.

Hoechst 33258 staining. Apoptotic morphological changes in the nuclear chromatin of cells were detected by Hoechst 33258 (Sigma) staining. AGS or HGC-27 cells were seeded on sterile cover glasses and washed with PBS and fixed with $4 \%$ paraformaldehyde for $10 \mathrm{~min}$, and then incubated with $50 \mu \mathrm{l}$ Hoechst 33258 staining solution for $10 \mathrm{~min}$. After three washes with PBS, the cells were viewed under a fluorescence microscope (Zeiss Axioskop; Zeiss, Oberkochen, Germany).

Western blot analysis. Cell lysates were subjected to polyacrylamide gel electrophoresis and blotted onto Immobilon-P polyvinylidene difluoride membranes (Millipore, Bedford, MA, USA). Caspase-3, caspase-8, caspase- $9, \beta$-catenin, cyclinD1 and C-myc antibodies were purchased from Santa Cruz Biotechnology, Inc. (Santa Cruz, CA, USA). $\beta$-actin antibody was purchased from GenScript, Inc. (Piscataway, NJ, USA), and the protein was detected using a HRP-conjugated secondary antibody and a Chemilucent ECL Detection System (Millipore, Billerica, MA, USA). The experiments were repeated at least twice with separately collected protein extracts.

miRNA analysis. Total RNA was extracted using the E.Z.N.A. ${ }^{\circledR}$ FFPE RNA Isolation kit (Omega) according to the manufacturer's instructions. For miRNA detection, $2 \mu \mathrm{g}$ of small RNA was reverse transcribed to cDNA using the miRNA First-Strand cDNA Synthesis kit (Invitrogen, USA) according to the manufacturer's instructions. Quantitative real-time PCR (qRT-PCR) analysis for miR-372, miR-21 and Let-7a was performed in triplicate using the SYBR-Green PCR Master Mix (Perkin-Elmer Applied Biosystems) according to the manufacturer's instructions. U6 RNA was used to normalize expression. Primers used were: U6 forward, 5'-CTCGCTTCGGCA GCACA-3'; Let-7a forward, 5'-GAGGTAGTAGGTTGT ATAGTTTAGAA-3'; miR-21 forward, 5'-GCTTATCAGAC TGATGTTGACTG-3', miR-372 forward, 5'-GCCCGCAA AGTGCTGCGAACAT-3'. All reverse primers were Uni-miR qPCR Primers obtained from the First-Strand cDNA Synthesis kit. Data analysis was performed using the $2^{-\Delta \Delta \mathrm{Ct}}$ method (27).

Immunofluorescence staining. The cells were plated overnight on coverslips on 12-well plates. AGS cells were pre-incubated with the proteosome inhibitor MG132 $(100 \mu \mathrm{M})$ for $5 \mathrm{~h}$ or the lysosome inhibitor $\mathrm{NH}_{4} \mathrm{Cl}(100 \mu \mathrm{M})$ for $5 \mathrm{~h}$, prior to caudatin $(50 \mu \mathrm{M})$ treatment for $24 \mathrm{~h}$. Cells were then fixed with paraformaldehyde for $15 \mathrm{~min}$, rinsed with PBS and incubated with $2 \%$ bovine serum albumin in PBS for $30 \mathrm{~min}$. The cells were then incubated overnight with the anti- $\beta$-catenin antibody. After washing with PBS, the cells were incubated with the Alexa Red-conjugated anti-mouse IgG secondary antibody (Molecular 

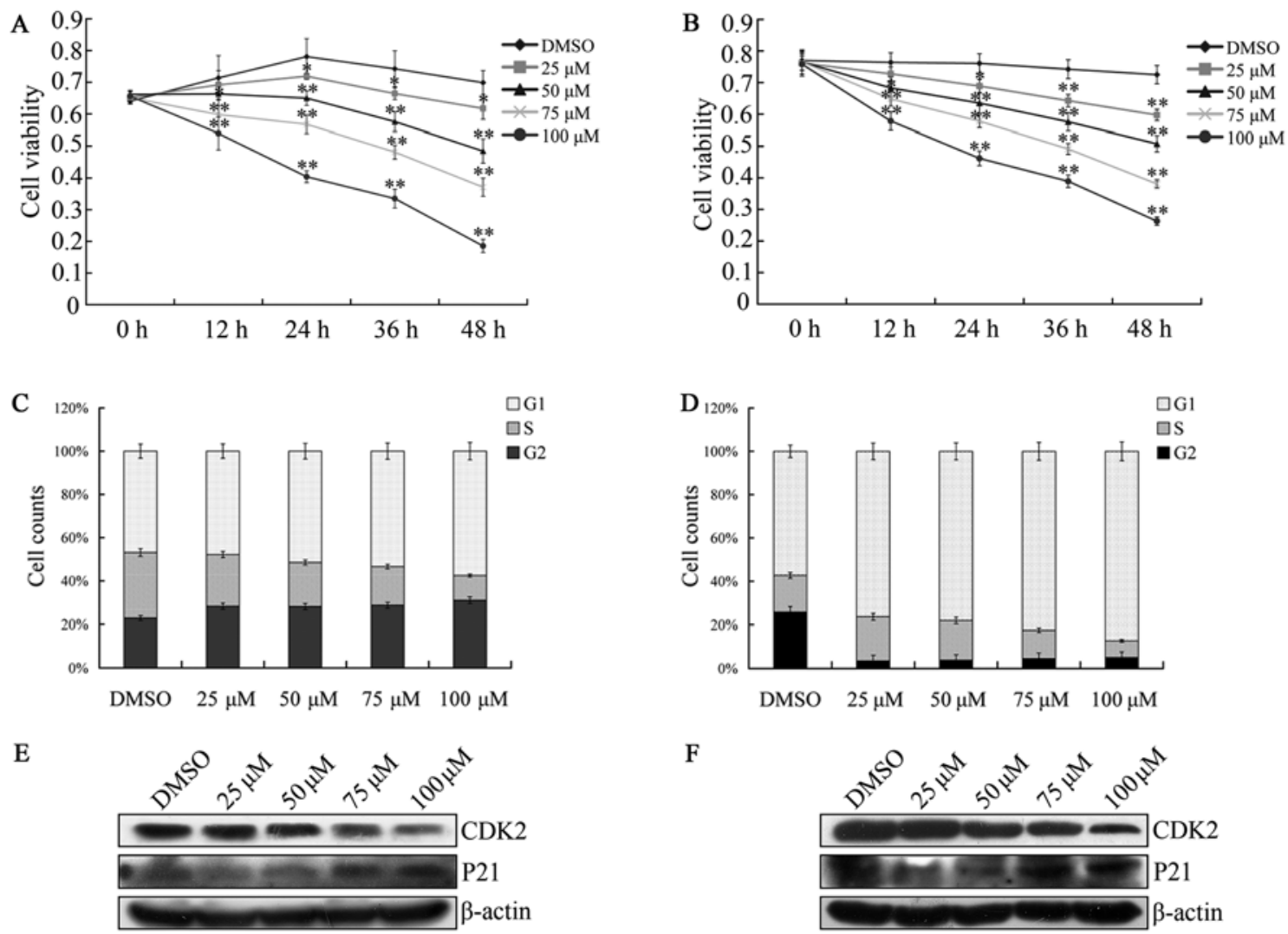

$\mathrm{F}$

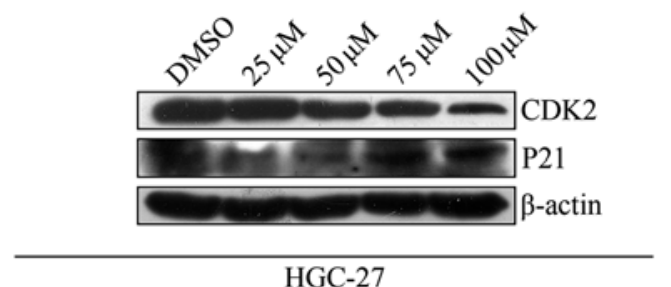

Figure 1. Caudatin inhibits the cell viability of human gastric cancer cell lines in a time- and dose-dependent manner. (A and B) Caudatin inhibits proliferation of AGS and HGC-27 cells. Cells were incubated with increasing doses of caudatin $(0-100 \mu \mathrm{M})$ for up to $48 \mathrm{~h}$ and analyzed for cell proliferation. Caudatin treatment resulted in a significant dose- and time-dependent decrease in cell proliferation in the two cell lines when compared with the untreated controls $(" \mathrm{P}<0.05$; $\left.{ }^{* *} \mathrm{P}<0.01\right)$. (C and D) Cell cycle analysis of caudatin-treated cells. AGS and HGC-27 cells were treated with caudatin $(0-100 \mu \mathrm{M})$ for $24 \mathrm{~h}$, and examined by flow cytometry following propidium iodide staining for DNA content. (E and F) Western blot analysis of cell lysates from AGS and HGC-27 cells for CDK2 and P21 protein expression levels. Caudatin treatment inhibited CDK2 protein expression and promoted P21 expression. The data represent the means \pm SD of three different experiments.

Probes). Hoechst 33258 was used to stain the cell nuclei. Cell images were observed under a fluorescence microscope.

Statistical analysis. All data are presented as means \pm standard deviation from at least three separate experiments. The differences among groups were analyzed using the double-sided Student's t-test, and statistical significance was determined at a P-value of $<0.05$.

\section{Results}

Caudatin inhibits gastric cancer cell growth. We determined the effect of caudatin on gastric cancer cell proliferation in the AGS and HGC-27 cells. Caudatin significantly suppressed the proliferation of the gastric cancer cell lines in a dose- and time-dependent manner. This anti-proliferative effect on tumor cells was observed for every 12 -h period following 5 treatments (DMSO or caudatin at $25,50,75,100 \mu \mathrm{M})($ Fig. $1 \mathrm{~A}$ and B). The $\mathrm{IC}_{50}$ values for caudatin in AGS cells at 12, 24, 36 and $48 \mathrm{~h}$ were $180.31,109.97,86.45$ and $54.92 \mu \mathrm{mol} / 1$, respectively. The $\mathrm{IC}_{50}$ values for caudatin in HGC-27 cells at $12,24,36$ and $48 \mathrm{~h}$ were $200.06,129.24,102.88$ and $65.98 \mu \mathrm{mol} / 1$, respectively. Of note, the MTT assay that is used to determine cell prolif- eration does not directly distinguish between induction of cell death or prevention of cell division; however, the result is clear that caudatin significantly suppressed the proliferation of the gastric cancer cell lines. Cell proliferation is also controlled by the progression of the cell cycle. Our results showed the treatment with caudatin distinctly inhibited the cell cycle of AGS and HGC-27 cells at the G1/S-phase transition (Fig. 1C and D). $\mathrm{CDK} 2$ is one of the cell cycle regulatory protein that regulates the G1 to S-phase transition of the cell cycle and functions as a cofactor for several transcription factors in numerous cell lines (28). Contrarily, P21 can suppress CDK2 and block the G1 to S-phase transition. In agreement with this theory, western blotting demonstrated that caudatin significantly downregulated CDK2 protein levels in a dose-dependent manner in the AGS and HGC-27 cells. In contrast, the expression of the P21 protein was upregulated distinctly after caudatin treatment (Fig. 1E and F). These results demonstrated that caudatin inhibited the growth of gastric cancer cells in a dose- and time-dependent manner.

Caudatin induces gastric cancer cell death by apoptosis. Flow cytometric analysis indicated the induction of apoptosis by caudatin occurred in AGS and HGC-27 cells. Caudatin treat- 


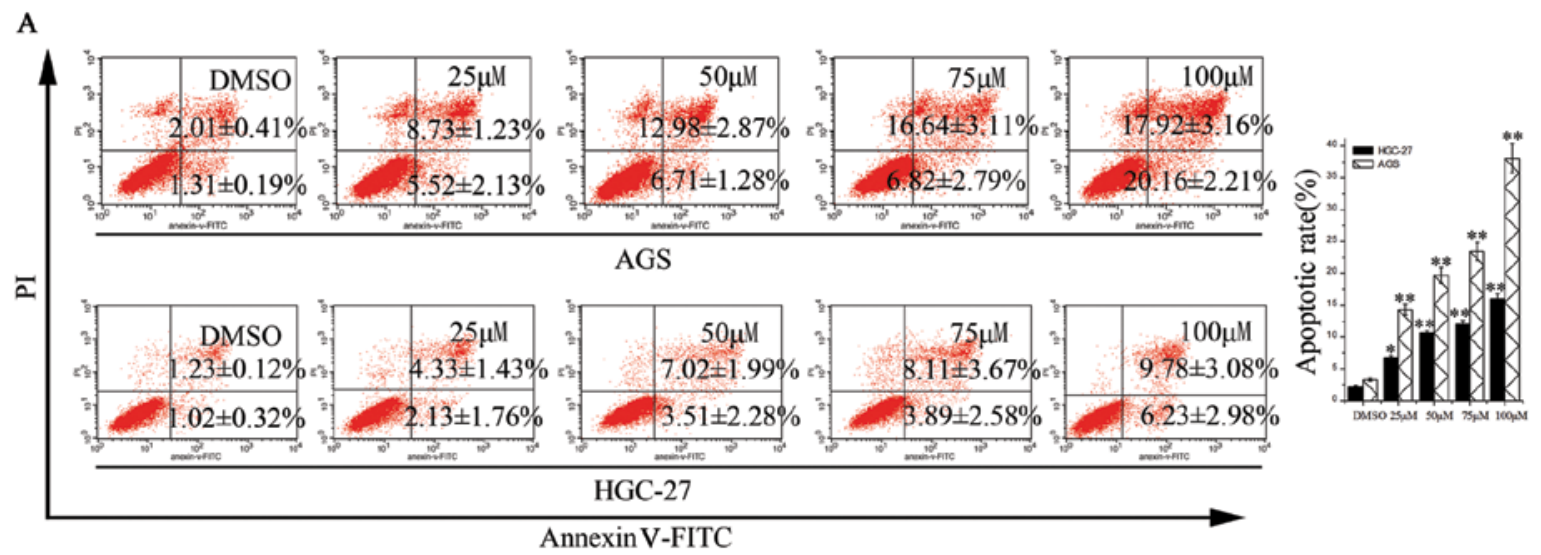

B
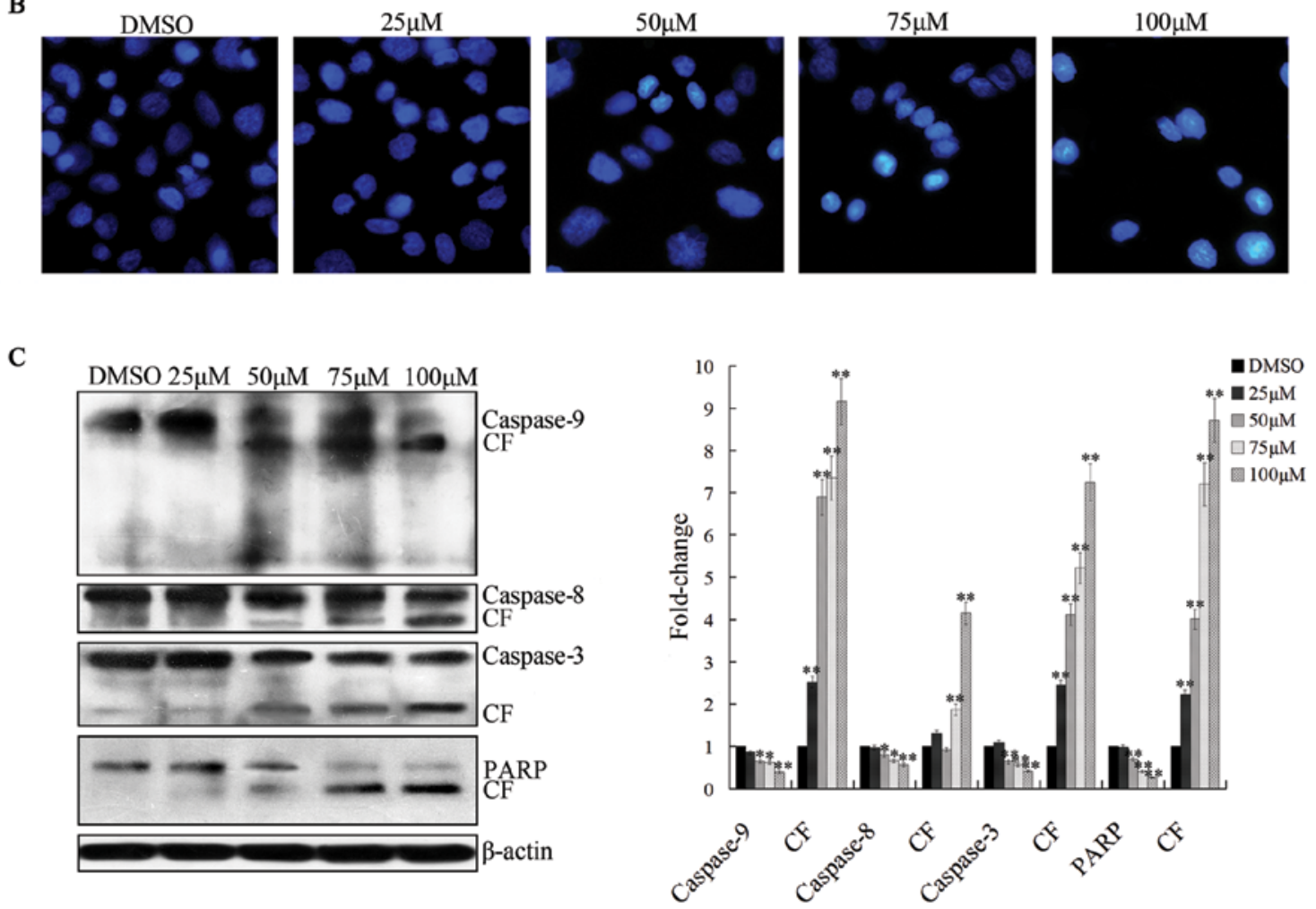

Figure 2. Caudatin activates the apoptotic pathway in gastric cancer cells. (A) AGS and HGC-27 cells were treated with different concentrations of caudatin for $24 \mathrm{~h}$, and then analyzed for apoptosis by flow cytometry for Annexin V and PI staining. Numbers in the respective quadrant profiles indicate the percentage of apoptotic cells. (B) Morphology of apoptotic cell nuclei was observed by Hoechst staining using a fluorescence microscope (magnification, $\mathrm{x} 40$ ). The control group was treated with DMSO. (C) The AGS cells were treated with different concentrations of caudatin. Western blot analysis revealed the cleavage of caspase-3, $-8,-9$ and PARP in response to various concentrations of caudatin. The data represent the means $\pm \mathrm{SD}$ of three different experiments. ${ }^{*} \mathrm{P}<0.05 ;{ }^{* *} \mathrm{P}<0.01$.

ment resulted in a substantial increase in Annexin V-positive cells (Fig. 2A), indicating induction of cell death via an apoptotic pathway. Notably, the AGS cells presented a higher apoptosis rate than the HGC-27 cells. Hochest 33258 staining indicated that typical apoptotic cell morphology, such as the formation of apoptotic bodies, appeared after the AGS cells were treated for $24 \mathrm{~h}$ with different concentrations $(25-100 \mu \mathrm{M})$ of caudatin, while the control cells did not obviously show evident apoptotic morphological changes (Fig. 2B). We confirmed this observation by evaluating the expression of PARP and caspase- $3,-8$ and -9 , as proteolytic cleavage and subsequent activation of these molecules activate apoptotic pathways. The expression level of full-length caspase-3, -8 and -9 was decreased, suggesting cleavage and activation of the caspase pathway. Moreover, cleaved products of caspase-3, -8 or -9 were also detectable. Furthermore, PARP, which is the prime marker for caspasedependent apoptosis, showed cleavage in a dose-dependent manner (Fig. 2C). These data suggest that caudatin is a potent inducer of apoptosis in gastric cancer cells, and the apoptosis involves the caspase-related pathway.

Caudatin reduces $\beta$-catenin expression in a dose-dependent manner. Deregulation of the Wnt/ $\beta$-catenin pathway has been shown in gastric cancer. As a modulator of the Wnt signaling pathway, $\beta$-catenin functions as a transcription factor that is translocated into the nucleus where it binds with the TCF 

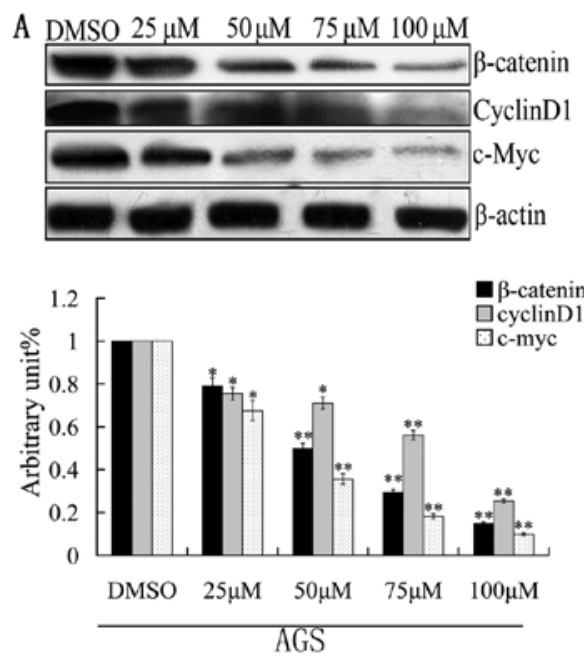

B
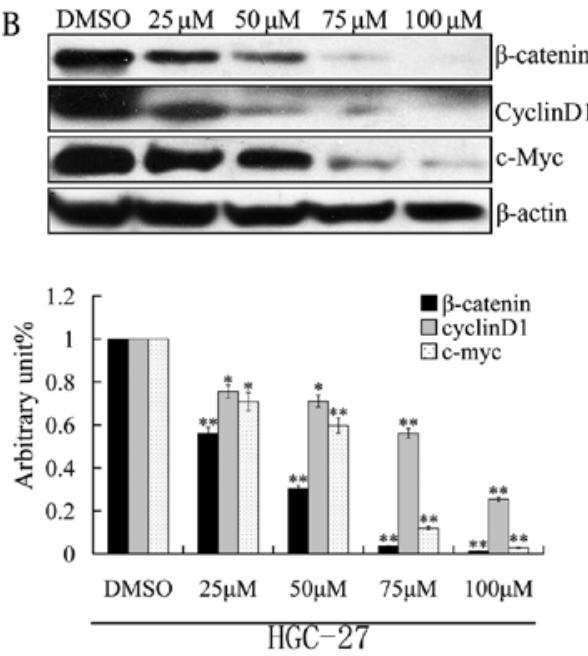

Figure 3. Caudatin inhibits $\beta$-catenin signaling and its downstream target proteins in gastric cancer cells. Western blot analysis of lysates following caudatin treatment showed a significant decrease in $\beta$-catenin and the protein levels of its target genes cyclinD1 and c-myc in (A) AGS and (B) HGC-27 cells. Data represent the average of three independent runs.
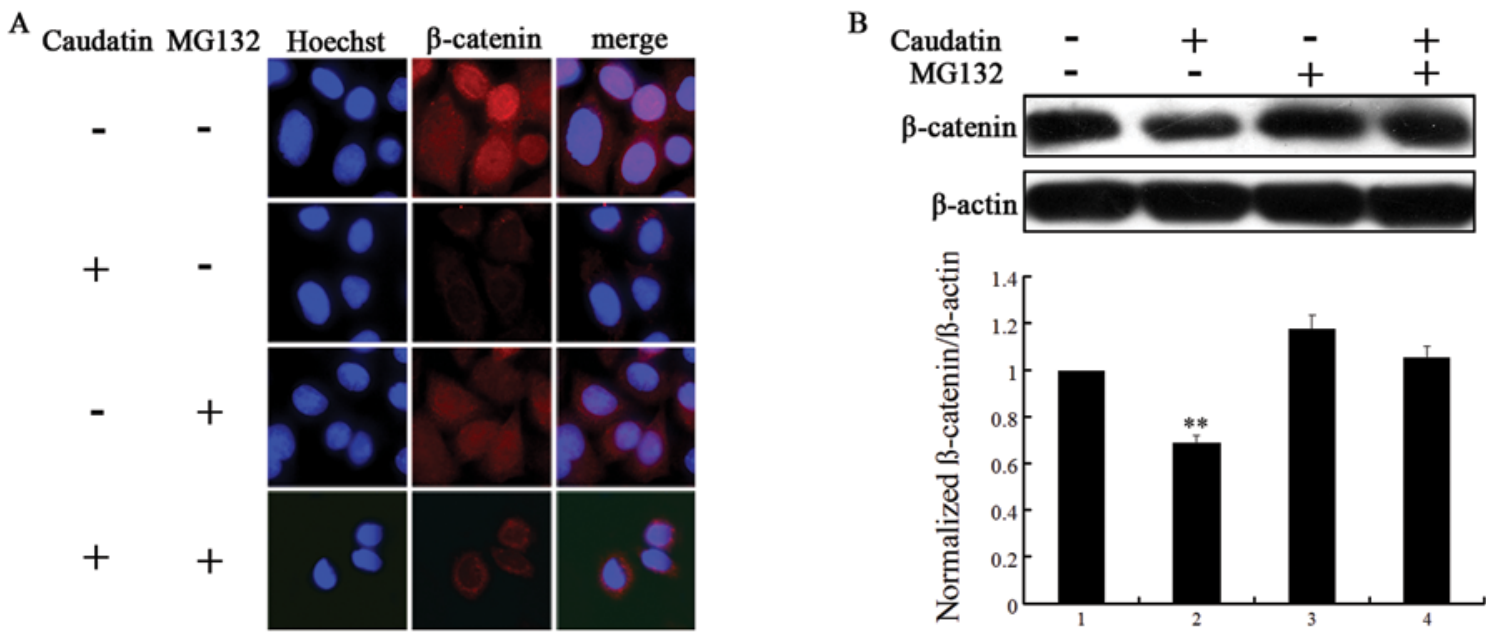

Figure 4. Caudatin induces proteosome-mediated degradation of $\beta$-catenin. (A) Immunocytochemical staining for $\beta$-catenin expression (magnification, $x 100$ ). Data represent the average of three independent runs; bars, mean \pm standard error (SEM). (B) Anti- $\beta$-catenin western blotting of whole cell extracts incubated with the MG132 proteosome inhibitor $(100 \mu \mathrm{M})$ for $5 \mathrm{~h}$, prior to caudatin $(50 \mu \mathrm{M})$ treatment for $24 \mathrm{~h} .{ }^{* * *} \mathrm{P}<0.01$.

transcription factor. To assess whether caudatin affects the expression of $\beta$-catenin, AGS and HGC-27 cells were treated with various concentrations of caudatin for $24 \mathrm{~h}$. Western blotting results indicated that caudatin significantly downregulated the expression of $\beta$-catenin in the AGS and HGC-27 cells. Inhibition of the level of cyclinD1, a downstream protein of $\beta$-catenin (29), was also observed following treatment with caudatin. This result supports the above conclusion that caudatin suppresses G1 to S-phase transition of the cell cycle in AGS and HGC-27 cells. In addition, the protein level of c-MYC (30), which is an important downstream regulatory gene of $\beta$-catenin, also was decreased (Fig. 3). These results suggest that caudatin blocks the $\mathrm{Wnt} / \beta$-catenin pathway by reducing the $\beta$-catenin protein level.

Caudatin induces proteasome-mediated degradation of $\beta$-catenin. The proteosome and lysosome pathways are two main degradation mechanisms of protein (31). To verify which pattern drives $\beta$-catenin into degradation, we pre-incubated AGS cells with the proteosome inhibitor MG132 $(100 \mu \mathrm{M})$ for $5 \mathrm{~h}$ or the lysosome inhibitor $\mathrm{NH}_{4} \mathrm{Cl}(100 \mu \mathrm{M})$ for $5 \mathrm{~h}$, prior to caudatin $(50 \mu \mathrm{M})$ treatment for $24 \mathrm{~h}$. Whole cell protein extracts were prepared and evaluated for $\beta$-catenin level by western blotting. As shown in Fig. 4B, the protein level of $\beta$-catenin was blocked in the presence of MG132. However, when AGS cells were pre-treated with $\mathrm{NH}_{4} \mathrm{Cl}$, there was no change in $\beta$-catenin protein level (data not shown). This result was confirmed using immunocytochemical staining in AGS cells (Fig. 4A). Notably, AGS cells treated with DMSO revealed nuclear location of $\beta$-catenin. Following treatment with $50 \mu \mathrm{M}$ of caudatin, $\beta$-catenin was mainly located under the plasma membrane. These data suggest that caudatin treatment attenuates nuclear $\beta$-catenin signaling, which is known to play a significant role in cancer cell proliferation. 

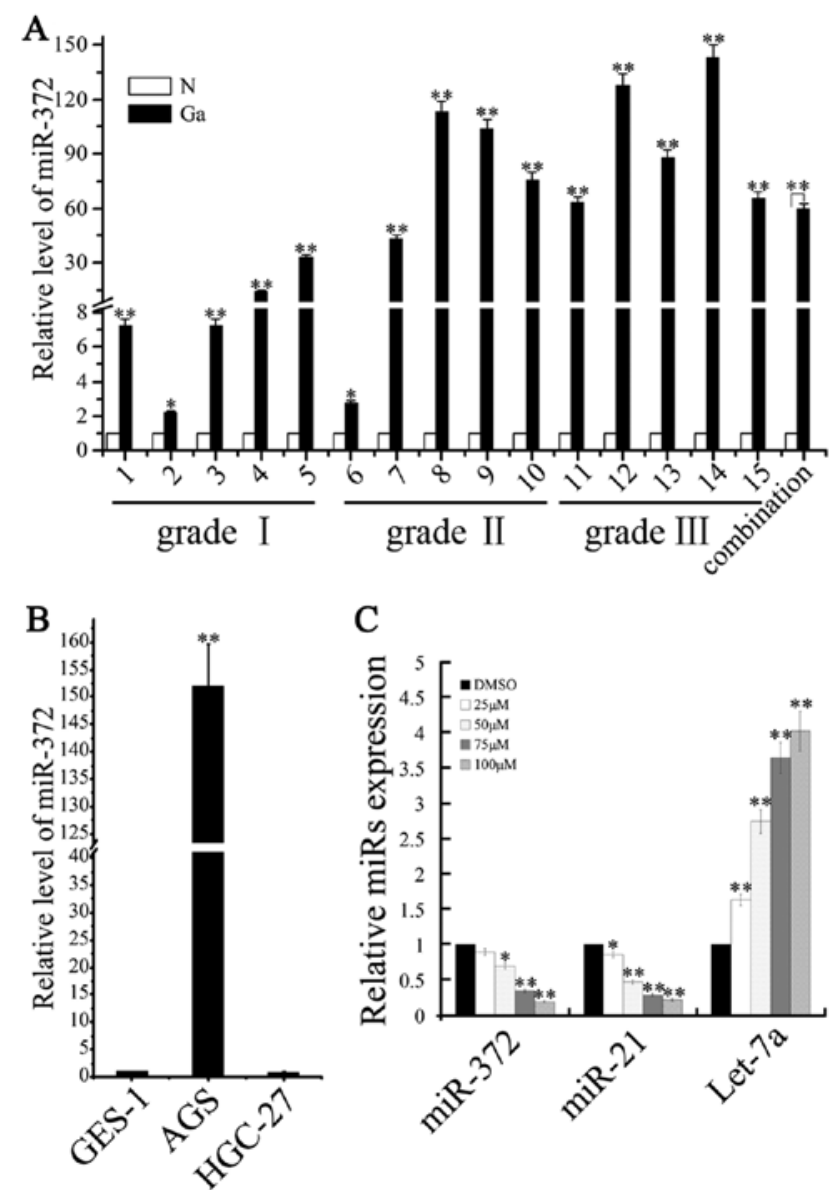

Figure 5. Caudatin treatment inhibits oncomir miRNA in AGS cells. (A) Real-time PCR analysis of miR-372 in gastric adenocarcinoma tissues and glioma tissues (Bars 1-5, grade I; 6-10, grade II; 11-15, grade III). N, normal tissue; Ga, gastric adenocarcinoma tissue. (B) Real-time PCR analysis of miR-372 in 3 gastric cancer cell lines (AGS, HGC-27, GES-1). RNA input was normalized by human U6 snRNA. (C) Real-time PCR analysis of total miRNA from AGS cells following treatment with various concentrations of caudatin for $24 \mathrm{~h}$. Caudatin treatment significantly inhibited oncomir miRNA expression, and upregulated tumor suppressor let-7a miRNA expression in AGS cells. The data represent the average of three independent runs; bars, mean \pm standard error $(\mathrm{SEM}) .{ }^{*} \mathrm{P}<0.05 ;{ }^{* *} \mathrm{P}<0.01$.

Caudatin inhibits oncomir miRNA and increases tumor suppressor miRNA in gastric cancer cells. Recently, we demonstrated that miRNA-372 maintains oncogene characteristics in AGS cells and may become a biomarker for gastric cancer progression, while Zhou et al (24) found that $\beta$-catenin transactivates the microRNA-371-373 cluster and modulates the Wnt/ $\beta$-catenin signaling pathway. To confirm the expression level of miR-372 in gastric adenocarcinoma tissue, total RNA was extracted from 15 gastric adenocarcinoma samples, consisting of 5 grade I, 5 grade II and 5 grade III gastric adenocarcinoma tissues, 15 matched normal tissues and 3 human gastric carcinoma cell lines. qRT-PCR was performed to analyze the expression profile. As shown in Fig. 5A, we found that miR-372 had higher expression levels in the gastric adenocarcinoma tissue when compared with the matched normal tissue. Moreover, miR-372 also showed abnormal upregulation in the adenocarcinoma cancer cells (AGS cells) (Fig. 5B). We next determined the effects of caudatin on oncomir miRNA and tumor suppressor miRNA in the gastric cancer cells.
Caudatin treatment significantly downregulated miR-372 and miR-21 expression while upregulating let-7a miRNA expression in a dose-dependent manner (Fig. 5C). Therefore, these findings reveal that the expression of miRNAs were altered after caudatin treated.

\section{Discussion}

Gastric cancer is increasing in incidence in many countries, and is among the top two causes of cancer-related mortality worldwide. It is estimated that 360,000 individuals succumb to gastric cancer each year in China. In the present study, we used gastric cancer cells as experimental material to confirm the antitumor effect of caudatin and to illustrate the underlying mechanisms of its anticancer activity. MTT assay was performed to quantify the effects of caudatin on AGS and HGC-27 cell growth inhibition. The data presented here showed that caudatin treatment resulted in a dose- and time-dependent inhibition of proliferation in gastric carcinoma cell lines. The $\mathrm{IC}_{50}$ values for caudatin in AGS cells at 12, 24, 36 and $48 \mathrm{~h}$ were $180.31,109.97,86.45$ and $54.92 \mu \mathrm{mol} / 1$, respectively. The $\mathrm{IC}_{50}$ values for caudatin in HGC-27 cells at 12, 24, 36 and $48 \mathrm{~h}$ were $200.06,129.24,102.88$ and $65.98 \mu \mathrm{mol} / 1$, respectively. The cell growth inhibition induced by many cancer chemopreventive agents is correlated with perturbations in the cell cycle progression. Thus, we determined the effect of caudatin on cell cycle distribution to gain insights into the mechanism of its cell growth suppression. Exposure of AGS and HGC-27 cells to caudatin resulted in the accumulation of the G0/G1 cell fraction in a concentration-dependent manner, which was accompanied by a decrease in the percentage of S-phase cells. $\mathrm{CDK} 2$ is one the cell cycle regulatory proteins that regulates the G1 to S-phase transition of the cell cycle and functions as a cofactor for several transcription factors in numerous cell lines. Contrarily, P21 can suppress CDK2 and block G1 to S-phase transition. In agreement with this theory (32), western blot analyses demonstrated that caudatin significantly downregulated CDK2 protein levels in a dose-dependent manner in the AGS and HGC-27 cells. In addition, p21, which plays a crucial role in the regulation of $\mathrm{G} 1$ to $\mathrm{S}$-phase transition by a $\mathrm{p} 53$-independent pathway, was also triggered by caudatin in gastric cancer cells. The expression of P21 protein was distinctly upregulated after caudatin treatment in the gastric carcinoma cell lines. Similar caudatin-induced G0/G1 arrest has also been shown to occur in other cancer cell types, including hepatic and lung cancers.

In addition to inappropriate growth signals, many cancer cells lose their ability to undergo apoptosis. Disturbances in apoptosis are important for the development of cancer. Therefore, the killing of tumors through induction of apoptosis has been recognized as a novel strategy for the identification of anticancer drugs. In order to distinguish that the cell death caused by caudatin is due to apoptosis or necrosis, Annexin V/PI double-labeling analysis was performed which enables further distinction of necrotic/late apoptotic (Annexin $\mathrm{V}^{+} / \mathrm{PI}^{+}$) and early apoptotic (Annexin $\mathrm{V}^{+} / \mathrm{PI}^{-}$) cells. Our results showed that treatment of AGS and HGC-27 cells with caudatin resulted in a time-dependent increase in the numbers of both early apoptotic and late apoptotic/necrotic cells. Hoechst staining showed that the typical morphological changes attributed to apoptosis, such as formation of apoptotic 
bodies, appeared after the cells were treated for $24 \mathrm{~h}$ with 25-100 $\mu \mathrm{M}$ caudatin, whereas the control cells did not show evident apoptotic morphological changes. Apoptosis may occur via a mitochondrial-dependent intrinsic pathway or a death receptor-mediated extrinsic pathway. Caspases, a family of cysteine proteases, are synthesized as inactive pro-enzymes which are processed to an active form in cells undergoing apoptosis. Caspase- 3 is situated at a pivotal junction in the apoptotic pathway and is considered to be the most important of the executioner caspases. Caspase- 3 is expressed in almost all types of cells as an inactive pro-enzyme and is activated by any of the initiator caspases (caspase-8, -9, or -10) and subsequently cleaves various specific substrates. Once activated, initiator caspases cleave and activate effector caspases, which in turn cleave a variety of cellular protein substrates, ultimately leading to cell apoptosis (33). In an attempt to identify the apoptotic signaling pathway induced by caudatin, we evaluated the cleavage of caspase-3, -8, -9 and PARP in AGS cells by western blot assay. Mechanistic studies showed that caudatin-induced cell apoptosis was accompanied by significant activation of caspase-3, -8 and -9 , and subsequent upregulated cleavage of PARP (Fig. 2).

The $\beta$-catenin pathway is known to be disrupted in a variety of cancers, including gastric cancer. It has also been shown that $\beta$-catenin activity also inhibits apoptosis in cancer cells. Activation of the $\beta$-catenin signaling pathway leads to nuclear localization of $\beta$-catenin which interacts with the TCF transcription factor and modulates the expression of a wide range of proto-oncogenes. Therefore, we hypothesized that caudatin exerts its effects through the modulation of the expression of $\beta$-catenin. In the present study, we found that caudatin downregulated the $\mathrm{Wnt} / \beta$-catenin signaling pathway through the inhibition of $\beta$-catenin in gastric cancer cells. We also found that caudatin treatment led to an $\sim 50 \%$ reduction in $\beta$-catenin, cyclinD1 and c-MYC protein levels (Fig. 3). These data suggest that caudatin-mediated cell growth inhibition and induction of cell apoptosis are partly mediated via inactivation of $\mathrm{Wnt} / \beta$-catenin activity, which is known to play a significant role in cancer cell proliferation. To further elucidate the pathway by which caudatin activates $\beta$-catenin degradation, we assessed the effects of the proteosome inhibitor MG132 or the lysosome pathway inhibitor $\mathrm{NH}_{4} \mathrm{Cl}$ in caudatin-treated AGS cells. We found that caudatin induced a reduction in $\beta$-catenin only via the proteosome pathway. As shown in Fig. 4, $\beta$-catenin was much more rapidly degraded in cells treated with caudatin than in cells treated with DMSO. However, treatment with the proteasomal inhibitor MG132 efficiently rescued the protein level of $\beta$-catenin, indicating that $\beta$-catenin was degraded in an ubiquitin-proteasome-dependent degradation manner. These results suggest that caudatin can increase the proteasome-dependent degradation of $\beta$-catenin. However, $\mathrm{Wnt} / \beta$-catenin is not the only pathway active in gastric cancer. It is thus important to determine whether caudatin is equally potent in inhibiting other signal transduction pathways.

microRNAs regulate multiple coding genes which are associated with tumor growth. Thus, assessment of specific miRNA expression is useful for predicting disease outcome. We recently demonstrated that miR-372 was abnormally upregulated in AGS cells and may be a biomarker for gastric adenocarcinoma. miR-372 can also act as an oncogene and promote the growth of testicular germ cells or AGS cells by targeting the LATS2 or TNFAIP1 gene $(34,35)$. Zhou et al $(24)$ demonstrated that $\beta$-catenin transactivates the microRNA-371-373 cluster. In the present study, this finding was further confirmed by detecting the miR-372 expression in 15 pairs of gastric adenocarcinoma tissues. The results showed that expression of miR-372 was upregulated in both gastric cancer tissues and AGS cells when compared to normal gastric tissues. Previous studies identified the altered expression of miR-21 in gastric cancer cells. Interestingly, miR-21 was previously shown to additionally target the tumor suppressor PTEN, RECK and PDCD4 and induce tumorgenesis (36), suggesting a general role for miR-21 in tumor progression. miR-21 can also inhibit apoptosis in breast cancer cells by regulating Bcl-2 expression (37). Let-7a is a tumor suppressor miRNA and is downregulated in many cancers. Let-7a decreased cell proliferation and migration of glioblastoma and reduced tumor size in a xenograft model. A recent study demonstrated that the let-7 family inhibits the proliferation of human gastric cancer cells by targetting HMGA2 (38). It would be interesting to determine whether caudatin affects the expression of these genes in gastric cancer cells. Caudatin inhibits oncomir miRNA and increases tumor suppressor miRNA in gastric cancer cells. Our result showed that caudatin treatment significantly reduced miR-372 and miR-21 expression while inducing let-7a in AGS cells. There is also an important link between caudatin, microRNAs and $\beta$-catenin regulation.

Taken together, an in vitro model was successfully established to evaluate the possible mechanism of caudatin in the therapy of gastric cancer. Our preliminary observations indicate that caudatin arrests proliferation of human gastric carcinoma cell lines and induces cell apoptosis, at least partially, via the caspase-dependent apoptotic pathway and the $\mathrm{Wnt} / \beta$-catenin signaling pathway. Thus, targeting $\beta$-catenin may be an effective strategy to control tissue destruction in gastric cancer patients. Caudatin seems to have multiple molecular targets and shows enhanced potency in various cancer cell lines. Thus, caudatin may be a strong candidate for therapeutic applications for gastric cancer as well as other types of cancer.

\section{Acknowledgements}

This study was supported by the National Natural Science Foundation of China (grant nos. 81071696, 31071150 and 81071656), the Project of Hunan Provincial Masters Innovation Fund (CX2012B227), Project of Chang Sha Science and Technology Plan (K1109006-31 and K1207014-31) and Hunan Normal University Student Innovation (2012042).

\section{References}

1. Catalano V, Labianca R, Beretta GD, Gatta G, de Braud F and Van Cutsem E: Gastric cancer. Crit Rev Oncol Hematol 71: 127-164, 2009.

2. Nakajima T: Gastric cancer treatment guidelines in Japan. Gastric Cancer 5: 1-5, 2002.

3. Han J, Jiao D, Cao J, Fing P, Liu X and Zhuang Y: Effects of the extract from semen viticis negundo with acetoactate on human gastric carcinoma SGC-7901 cells in vitro and in vivo. Chinese Pharmacological Bulletin 12: 029, 2008.

4. Yang NI and Yi-ping YE: Distribution of C_(21) steroidal glycosides in plants of Asclepiadaceae and their pharmacological activities. Chinese Traditional and Herbal Drugs 1: 047, 2010 (In Chinese). 
5. Yao N, Gu X and Li Y: Effects of three C21 steroidal saponins from Cynanchum auriculatum on cell growth and cell cycle of human lung cancer A549 cells. Zhongguo Zhong Yao Za Zhi 34: 1418-1421, 2009 (In Chinese).

6. Fei HR, Chen HL, Xiao T, Chen G and Wang FZ: Caudatin induces cell cycle arrest and caspase-dependent apoptosis in HepG2 cell. Mol Biol Rep 39: 131-138, 2012.

7. Peng YR, Li YB, Liu XD, Zhang JF and Duan JA: Apoptosis induced by caudatin in human hepatoma cell line SMMC7721. Chin J Nat Med 6: 210-213, 2008.

8. Wang LJ, Geng CA, Ma YB, et al: Design, synthesis, and molecular hybrids of caudatin and cinnamic acids as novel antihepatitis B virus agents. Eur J Med Chem 54: 352-365, 2012.

9. Peng YR, Ding YF, Wei YJ, Shu B, Li YB and Liu XD: Caudatin2,6 -dideoxy-3-O-methy- $\beta$-D-cymaropyranoside 1 induced apoptosis through caspase 3-dependent pathway in human hepatoma cell line SMMC7721. Phytother Res 25: 631-637, 2011.

10. MacDonald BT, Tamai K and He X: Wnt/ $/$-catenin signaling: components, mechanisms, and diseases. Dev Cell 17: 9-26, 2009.

11. Naito AT, Shiojima I and Komuro I: Wnt signaling and agingrelated heart disorders. Circ Res 107: 1295-1303, 2010.

12. Lee E, Salic A, Krüger R, Heinrich R and Kirschner MW: The roles of APC and Axin derived from experimental and theoretical analysis of the Wnt pathway. PLos Biol 1: E10, 2003.

13. Liu C, Li Y, Semenov M, et al: Control of $\beta$-catenin phosphorylation/degradation by a dual-kinase mechanism. Cell 108: 837-847, 2002.

14. Clements WM, Wang J, Sarnaik A, et al: $\beta$-Catenin mutation is a frequent cause of Wnt pathway activation in gastric cancer. Cancer Res 62: 3503-3506, 2002.

15. Huiping C, Kristjansdottir S, Jonasson JG, Magnusson J, Egilsson V and Ingvarsson S: Alterations of E-cadherin and $\beta$-catenin in gastric cancer. BMC Cancer 1: 16, 2001.

16. Luu HH, Zhang R, Haydon RC, et al: Wnt/ $\beta$-catenin signaling pathway as novel cancer drug targets. Curr Cancer Drug Targets 4: 653-671, 2004.

17. Bartel DP: MicroRNAs: genomics, biogenesis, mechanism, and function. Cell 116: 281-297, 2004

18. Carleton M, Cleary MA and Linsley PS: MicroRNAs and cell cycle regulation. Cell Cycle 6: 2127-2132, 2007.

19. Wienholds E and Plasterk RH: MicroRNA function in animal development. FEBS Lett 579: 5911-5922, 2005.

20. Xiao B, Guo J, Miao Y, et al: Detection of miR-106a in gastric carcinoma and its clinical significance. Clin Chim Acta 400: 97-102, 2009.

21. Zhang Z, Li Z, Gao C, et al: miR-21 plays a pivotal role in gastric cancer pathogenesis and progression. Lab Invest 88: 1358-1366, 2008 .
22. Belair C, Baud J, Chabas S, et al: Helicobacter pylori interferes with an embryonic stem cell microRNA cluster to block cell cycle progression. Silence 2: 7, 2011.

23. Zhou C, Li X, Zhang X, et al: microRNA-372 maintains oncogene characteristics by targeting TNFAIP1 and affects $\mathrm{NF} \kappa \mathrm{B}$ signaling in human gastric carcinoma cells. Int J Oncol 42: 635-642, 2012

24. Zhou AD, Diao LT, Xu H, et al: $\beta$-Catenin/LEF1 transactivates the microRNA-371-373 cluster that modulates the Wnt/ $\beta$-cateninsignaling pathway. Oncogene 31: 2968-2978, 2011

25. Lee ST, Chu K, Oh HJ, et al: Let-7 microRNA inhibits the proliferation of human glioblastoma cells. J Neurooncol 102: 19-24, 2011.

26. Motoyama K, Inoue H, Nakamura Y, Uetake H, Sugihara K and Mori M: Clinical significance of high mobility group A2 in human gastric cancer and its relationship to let-7 microRNA family. Clin Cancer Res 14: 2334-2340, 2008.

27. Livak KJ and Schmittgen TD: Analysis of relative gene expression data using real-time quantitative PCR and the $2^{-\Delta \Delta C T}$ method. Methods 25: 402-408, 2001.

28. Tsai LH, Lees E, Faha B, Harlow E and Riabowol K: The cdk2 kinase is required for the G1-to-S transition in mammalian cells. Oncogene 8: 1593-1602, 1993.

29. Tetsu O and McCormick F: $\beta$-Catenin regulates expression of cyclinD1 in colon carcinoma cells. Nature 398: 422-426, 1999.

30. He TC, Sparks AB, Rago C, et al: Identification of c-MYC as a target of the APC pathway. Science 281: 1509-1512, 1998.

31. Ciechanover A: Proteolysis: from the lysosome to ubiquitin and the proteasome. Nat Rev Mol Cell Biol 6: 79-87, 2005.

32. Harper JW, Elledge SJ, Keyomarsi K, et al: Inhibition of cyclindependent kinases by p21. Mol Biol Cell 6: 387-400, 1995.

33. Cohen GM: Caspases: the executioners of apoptosis. Biochem J 326: 1-16, 1997.

34. Cho WJ, Shin JM, Kim JS, et al: miR-372 regulates cell cycle and apoptosis of ags human gastric cancer cell line through direct regulation of LATS2. Mol Cells 28: 521-527, 2009.

35. Voorhoeve PM, le Sage C, Schrier M, et al: A genetic screen implicates miRNA-372 and miRNA-373 as oncogenes in testicular germ cell tumors. Cell 124: 1169-1181, 2006.

36. Asangani IA, Rasheed SA, Nikolova DA, et al: MicroRNA-21 (miR-21) post-transcriptionally downregulates tumor suppressor Pdcd 4 and stimulates invasion, intravasation and metastasis in colorectal cancer. Oncogene 27: 2128-2136, 2008.

37. Si M, Zhu S, Wu H, Lu Z, Wu F and Mo YY: miR-21-mediated tumor growth. Oncogene 26: 2799-2803, 2007.

38. Lee YS and Dutta A: The tumor suppressor microRNA let-7 represses the HMGA2 oncogene. Genes Dev 21: 1025-1030, 2007. 